\title{
Low Voltage SEM and Correlative Microscopy to Analyze Delicate Biological Material
}

Heide Schatten

University of Missouri, Department of Veterinary Pathobiology, Columbia, MO 65211, USA

Advances in correlative microscopy have made it possible to compare and analyze samples on light and electron microscopy levels. For delicate isolated biological material the capabilities provided by low voltage field emission scanning electron microscopy (LVFESEM) have particularly been helpful to allow comparison analysis of structures such as the isolated mitotic apparatus [1-9].

Our research has been focused on microtubule organization and microtubule functions in cells during early reproduction in which the sperm triggers assembly of microtubule formations including the sperm aster, zygote aster, and the resulting mitotic apparatus. Centrosome organization and dynamics, and the interactions with centrioles are further revealed by LVFESEM and correlated with immunofluorescence images. While conventional TEM has not revealed sufficient detail of centrosome structure, the FESEM has given us the first close insights into microtubule-centrosome interactions in the sperm aster, and its dynamic reorganization and remodeling into the mitotic apparatus during the first embryonic cell in which centrosomes play critical roles, as will be discussed in the presentation.

\section{References}

[1] Schatten, H. (2008). High-Resolution, Low Voltage, Field-Emission Scanning Electron Microscopy (HRLVFESEM) Applications for Cell Biology and Specimen Preparation Protocols. In: Biological Low-Voltage Scanning Electron Microscopy. Edited by H. Schatten and J. Pawley. Springer, New York, Heidelberg, Berlin.

[2] Schatten, H. (2008). The mammalian centrosome and its functional significance. Histochem. Cell Biol. 129:667-686.

[3] Schatten, H., and Sun, Q-Y. (2009). The functional significance of centrosomes in mammalian meiosis, fertilization, development, nuclear transfer, and stem cell differentiation. Environ Mol Mutagen 50(8): 620-636.

[4] Schatten, H., and Sun, Q-Y. (2010). The role of centrosomes in fertilization, cell division and establishment of asymmetry during embryo development. Seminars in Cell and Developmental Biology 21:174-184.

[5] Schatten, H., and Sun, Q-Y. (2011). New insights into the role of centrosomes in mammalian fertilisation and implications for ART. Reproduction 142:793-801.

[6] Schatten, H., Rawe, V.Y., and Sun, Q-Y. (2012). Cytoskeletal architecture of human oocytes with focus on centrosomes and their significant role in fertilization. In: Practical Manual of In Vitro Fertilization: Advanced Methods and Novel Devices, edited by Zsolt Peter Nagy, Alex C. Varghese, and Ashok Agarwal. Humana Press (Springer Science+Business Media, New York, USA). 
[7] Schatten H, Sun Q-Y. (2012). Nuclear-centrosome relationships during fertilization, cell division, embryo development, and in somatic cell nuclear transfer (SCNT) embryos. In: The Centrosome; edited by Heide Schatten, Springer Science and Business Media, LLC (July 2012) [8] Schatten, H. and Sun, Q-Y. (2013). The role of the sperm centrosome in reproductive fitness. In: Paternal Influences on Human Reproductive Success, edited by Dr Douglas Carrell, published by Oxford University Press, 2013.

[9] Schatten, H. and Sun, Q-Y. (2014). "Posttranslationally modified tubulins and other cytoskeletal proteins: Their role in gametogenesis, oocyte maturation, fertilization and preimplantation embryo development. In Posttranslational Protein Modifications in the Reproductive System; Edited by Peter Sutovsky; published by Springer Science and Business Media
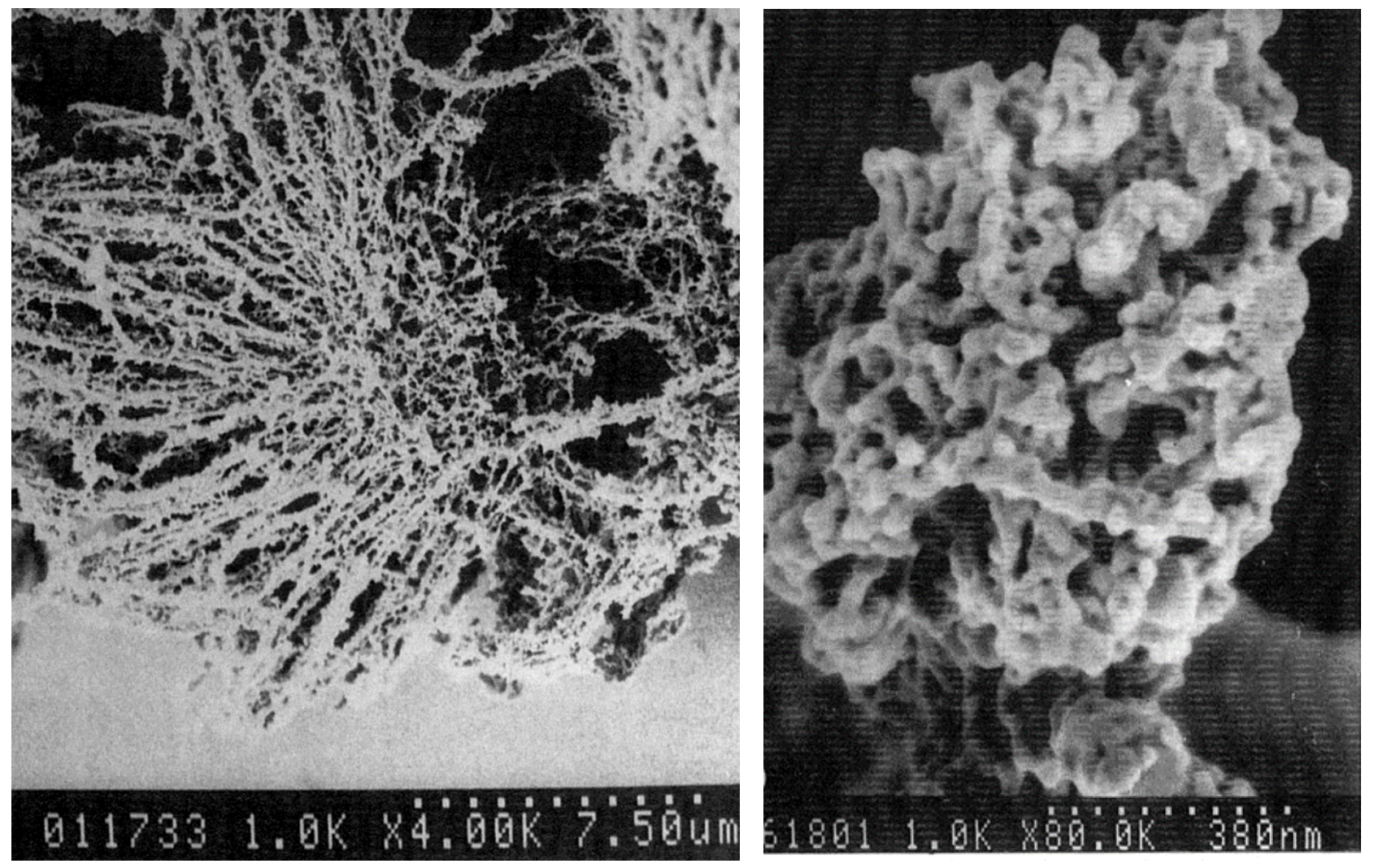

Figure 1: Left: LVFESEM of aster from isolated mitotic spindle in which microtubules emanate from a central core. Right: Isolated centrosomal material Modified from [1]. 